\title{
Physical activity and situational interest in mobile technology integrated physical education: A preliminary study
}

\author{
Xihe Zhu' ${ }^{1, *}$ and Loren A. Dragon ${ }^{2}$ \\ ${ }^{1}$ Darden College of Education, Old Dominion University, Norfolk, VA, United States; and ${ }^{2}$ Lynnhaven Middle School, \\ Virginia Beach, VA, United States
}

Copyright: (c) 2016 X. Zhu and L. A. Dragon. This is an open access article licensed under the Creative Commons Attribution License (http://creativecommons.org/licenses/by/4.0/).

\begin{abstract}
Background: Mobile technology permeates every aspect of student lives. The question is whether mobile technology integration can produce desirable effects in the gymnasium. Objective: This preliminary study aimed to investigate the effects of mobile technology integration on student situational interest and physical activity fluctuation in physical education lessons. Methods: Sixth grade students $(N=53)$ were randomly placed into either an experiment group by class that utilized mobile technology-integrated resources (iPad and applications), or a comparison group that did not utilize technology. Both groups received five identical physical education lessons. Student physical activity was tracked with accelerometers, and they completed the Situational Interest Scale at the end of each lesson. The researchers analyzed the data using descriptive statistics, correlation analysis and multivariate analysis of variance (MANOVA) with repeated measures. Results: Students in the experiment group reported significantly lower physical activity and situational interest than their counterparts in the comparison group. A group $\times$ lesson interaction suggested that student step/min steadily increased throughout the lessons in the experiment group while remaining relative stable in the comparison group. Conclusions: Mobile technologies such as iPad and applications with no direct physical activity prompt had little effect on increasing physical activity or situational interest in the short term. It is important to consider the classroom dynamics to realistically evaluate the constraints and strengths that mobile technology-integrated physical education lessons may pose in a traditional physical education environment.
\end{abstract}

Keywords: activity fluctuation, iPad application, middle school, adolescent, intervention

\section{Introduction}

In an age of high prevalence of childhood obesity, professional associations and government agencies advocate physical education to serve the interest of public health. The National Association for Sport and Physical Education (National Association for Sport and Physical Education, 2003) has maintained that a quality physical education program should not only offer sufficient opportunities for students to learn psychomotor skills, but it should also provide an engaging environment for effective physical activity accumulation. More specifically, the Centers for Disease Control and Prevention (Centers for Disease Control and Prevention, 2010) suggest that a quality physical education lesson

\footnotetext{
* Address for correspondence: Xihe Zhu, Department of $\mathrm{Hu}-$ man Movement Sciences, Darden College of Education, Old Dominion University, 2010 Student Rec. Center, Norfolk, VA 23529, United States. E-mail: x2zhu@odu.edu
}

should allocate at least $50 \%$ of its instructional time to moderate-to-vigorous physical activity (MVPA). To enhance physical activity accumulation, an interesting class environment would naturally be needed in physical education (Shen \& Chen, 2006).

Situational interest refers to a learner's appeal generated through interaction with a specific task or in a particular context. Situational interest is conceptualized as ephemeral but with immediate effects in engaging students (Hidi \& Renninger, 2006). Chen, Darst, and Pangrazi (1999) identified five sources of overall situational interest in physical education setting: attention demand, challenge, exploration opportunity, instant enjoyment and novelty. Shen and Chen (2006) found a moderate correlation between situational interest and the step count taken in physical education class $(r=.48)$. Recently, electronic technologies have been employed in physical education to promote both situational interest and physical activity (Sun, 2012). However, most of the studies only measured physical 
activity and/or situational interest once or twice, consequently failing to elucidate their fluctuation as a result of the technology applications. The purpose of this study was to investigate student situational interest and physical activity fluctuation through five mobile technology-integrated physical education lessons.

\section{Technology, physical activity, and interest in physical education}

A recent survey found that $39.4 \%$ of teachers had access to an iPad or Kindle with applications at school that can be utilized to integrate-technology into classrooms and physical education (Kervin, Verenikina, Jones, \& Beth, 2013). When teachers begin to integrate technology effectively into their physical education lessons, researchers can examine the effects of technology on student interest and engagement. Casey and Jones (2011) utilized digital video technology in an effort to increase the engagement of students who were categorized as having a low engagement level. The students were introduced to the technology and data was analyzed to determine the impact the video had on intellectual quality, quality learning environment and significance. The teacher believed that the students demonstrated a greater depth of knowledge of throwing and catching skills as a direct result of the use of technology (Casey \& Jones, 2011). The technology used in the research also gave students the knowledge they needed to critique others' performance in relation to the throwing and catching activity. This research was a positive step toward further engaging students in physical education lessons with technology-integrated activities.

In recent years, an array of technologies such as pedometers, smartphones, tablets (applications) virtual reality simulators, heart rate monitors, and Exergames (e.g., Dance Dance Revolution, and Sony Play Station games) are being used in physical education, sport, and physical activity to enhance engagement, pedagogy, and performance (Hall, 2012). Sun (2012) investigated the effect of Exergames on students' situational interest in physical education. Students participated in an Exergame unit and physical fitness unit and their physical activity and situational interest levels were tracked over a four week period. In this study, situational interest was measured by using student responses to the Situational Interest Scale (Chen et al., 1999) to determine the initial level of interest and retained level of interest. The Exergame unit was more effective than the fitness unit at engaging students' initial situational interest sources. However, these effects were not retained for all areas of situational interest by the end of the Exergame unit. Sun (2012) also suggested that the students did not meet recommendations for MVPA during the
Exergames unit as measured in units of Metabolic Equivalent of Task (MET) $(M=2.14, S D=0.65)$, but did meet the recommendations (> 3.0 MET) during the fitness unit reported in MET units $(M=4.1$, $S D=0.93)$.

\section{The current study}

The Exergames are not good representatives of mobile technologies. In fact, they are not mobile. To the researchers' knowledge, no empirical research has been reported to examine the integration of mobile technology in physical education. Therefore, in this study, the researchers investigated student interest and physical activity in technology-integrated physical education lessons to determine whether their situational interest and physical activity levels changed over time within the technology-integrated lessons. Based on the previous studies, it is hypothesized that (a) mobile technology would elevate student physical activity and situational interest, and (b) student physical activity and situational interest would remain relatively stable given the content are similar. This study would be the first to report the effects of mobile technology integration on physical activity and situational interest in physical education.

\section{Methods}

\section{Study design}

This preliminary study utilized a quasi-experimental design where participants were placed in the experiment or comparison group by class. Students in the experiment group participated in technology-integrated physical education lessons while students in the comparison group participated in the exact same lessons without the use of mobile technology. The study period lasted approximately two weeks, and the lesson activities took place every other day for a total of five lessons. Students' physical activity levels and situational interest levels were tracked during each of the research lessons. To ensure implementation fidelity, one researcher observed all the physical education classes during the research period to ensure that the lessons were being delivered consistently faithfully for each of the groups.

\section{Participants and research context}

A total of 53 sixth grade students from four independent classes participated in the study. Sixth grade students were chosen as the participants for the research because they had not been previously introduced to the technology-integrated physical education lessons. The participants' ages ranged from 10-12 years, $M=11.04$ $(S D=0.33)$. Overall, the sample included 38 females 
(71.70\%) and 15 males (28.30\%) and did not represent a gender-balanced population. Based on participant self-report, the sample was comprised of 7.54\% Asian, 9.43\% African American, 18.86\% Latino, 62.29\% Caucasian, and $1.88 \%$ other students. Table 1 shows the descriptive data for participant ethnicity, gender, and body mass index for the experiment and comparison groups.

The physical education classes were taught by four physical education specialists. Students attended daily physical education for 50 minute blocks; the first and last 8-10 minutes of each block were spent having students dress in the locker room. With dressing time accounted for, students were potentially active for a maximum of 30-34 minutes per class. Each of the teachers typically began their lessons with a warm up section. Students were then given instruction for the activities to be completed for the remainder of the lesson. At the end of the physical education class, students returned all equipment to the designated area, lined up based on gender, and were dismissed into the locker rooms. The school had two iPad carts for a total of 60 iPads available for use within the building to meet the student's needs during the technology-integrated physical education lessons.

\section{Variable and measures}

Situational interest

Student overall situational interest was measured using the Situational Interest Scale. The scale was developed by Chen et al. (1999) to measure students' situational interest in the physical education setting. The scale has 24 items, six subscales (four items each) measuring five sources of situational interest (attention demand, challenge, exploration intention, instant enjoyment, and novelty), and overall interest. The internal consistency coefficients, Cronbach's $\alpha$, are .78, .80, .90, .91, .90, and .95 for the five sources and overall situational interest (Chen et al., 1999). Because we did not intend to look at the fluctuation of the five sources, and the full scale (24 items) was lengthy for students to complete repetitively, we used only the subscale for overall situational interest (four items). These four items are: "it was fun for me to try what we were doing in the lesson"; "what we were doing in the lesson was really interesting"; "what we were doing looked really fun to me"; "what we were doing got my interest". The students respond to a Likert-type scale ranging from 5 (strongly agree) and 1 (strongly disagree) to assess their feelings toward the five lessons, at the end of each lesson.

\section{Physical activity}

Student physical activity levels were recorded using GT3X ActiGraph tri-axial accelerometers (ActiGraph, Pensacola, FL, USA). This small $(3.8 \times 3.7 \times 1.8 \mathrm{~cm})$, lightweight device $(27 \mathrm{~g})$ is often used in physical activity research (Sasaki, John, \& Freedson, 2011). The ActiGraph GT3X has been reported to generate estimate of energy expenditure correlated with oxygen consumption ( $r=.88$ ) and to classify the participants' MVPA correctly in $86 \%$ of cases, showing good validity (Hänggia, Phillips, \& Rowlands, 2013). The accelerometers were programed in $10 \mathrm{~s}$ epoch and $30 \mathrm{~Hz}$ for sampling frequency during initialization. Intensity cut-points (Freedson, Pober, \& Janz, 2005; Sasaki et al., 2011) were used to calculate minutes of MVPA (i.e., $\geq 3$ METs, 2,690 counts/min), light physical activity (i.e., 1.9-2.99 METs), and sedentary time (i.e., $<1.9 \mathrm{METs}$ ). The Freedson combination equation was used to estimate energy expenditure (Sasaki et al., 2011). During the research period, students wore programmed GT3X, tri-axial accelerometers immediately once they entered the gymnasium, and returned them right after the class dismissal.

\section{Procedures}

Following approval of the university institutional review board, the researchers obtained permission from the specific public school system. Prior to the commencement of research, parental consent and student assent

Table 1

Descriptive statistics of the sample for gender, ethnicity ( $n / \%)$, and body mass index $(M \pm S D)$

\begin{tabular}{lccc}
\hline Variable & Experiment group & Comparison group & Total \\
\hline Male & $7(25.93 \%)$ & $8(30.77 \%)$ & $15(28.30 \%)$ \\
Female & $20(74.07 \%)$ & $18(69.23 \%)$ & $38(71.70 \%)$ \\
Asian & $2(7.41 \%)$ & $2(7.69 \%)$ & $4(7.54 \%)$ \\
African American & $1(3.70 \%)$ & $4(15.38 \%)$ & $5(9.43 \%)$ \\
Latino & $10(37.04 \%)$ & $0(0.00 \%)$ & $10(18.86 \%)$ \\
Caucasian & $14(51.85 \%)$ & $19(73.09 \%)$ & $33(62.29 \%)$ \\
Other & $0(0.00 \%)$ & $1(3.84 \%)$ & $1(1.88 \%)$ \\
Body mass index $\left(\mathrm{kg} / \mathrm{m}^{2}\right)$ & $20.46 \pm 4.73$ & $20.22 \pm 4.96$ & $20.34 \pm 4.85$ \\
\hline
\end{tabular}


for voluntary participation were obtained. Students in the experiment group were familiarized with the accelerometers, QR codes (ISO 18004:2015; http://www. iso.org/iso/catalogue_detail.htm?csnumber $=62021)$, Instant Heart Rate Calculator (Azumio Inc., 2015; http://www.azumio.com/s/instantheartrate), and Edmodo (Edmodo Inc., 2016; http://www.edmodo. com) applications that would be utilized during the research phase. This process ensured that students were able to access the applications and perform the required fundamental actions of the technology-integrated lessons. Students in the comparison group were not acquainted with these applications other than how to pick up, use and return the accelerometer during the lesson.

For the experiment group, QR codes were scanned using the iPads and the directions for the physical activity that students were required to participate in appeared on the iPads. Directions from the QR code included the equipment used during the activity and how many repetitions of the activity the student was required to perform. The physical education teachers were available for assistance with activity directions and to troubleshoot any problems that arose during the technology-integrated lessons. Immediately after participating in the physical activity, students assessed their heart rate using the Instant Heart Rate Calculator. They utilized a digital data table via the Edmodo application to enter their heart rate and to analyze changes during the varying levels of physical activity.

The comparison group of students participated in the same five relative physical activity intensity, heart rate measures, energy expenditure and energy balance lessons, but without the technology-integrated resources. Students were given verbal directions for each of the fitness activities that they were required to participate by their physical education teachers. After participating in the lesson activities, students assessed their intensity levels through manual heart rate calculation, and recorded it onto a physical activity log with pencil and paper. Table 2 summarizes the major instructional differences and the lesson content and physical activities used for the experiment and comparison groups. The tasks in the fitness stations were balanced across the lessons such that the required physical activity intensity and duration was comparable among the lessons, if completed as stated in the lesson plans.

\section{Data analysis}

The physical activity and situational interest data were analyzed using descriptive and inferential statistics. To begin data analysis, descriptive statistics were used to describe the sample through the measures of central tendency and variability. A frequency analysis and tests of normality were conducted to determine whether the variables of interest and physical activity were normally distributed and whether extreme outliers needed to be removed from the sample. Step/min was computed to account for the small variation of lessons duration, using the total step count divided by the recorded lesson time. The data was further analyzed using a Pearson product-moment correlational analysis to identify any significant correlation between the two variables of physical activity and situational interest. Finally, inferential statistics were used to perform analysis of variance with repeated measure on the variables of situational interest and physical activity in both the technology-integrated physical education and comparison groups. We used SPSS (Version 21; IBM, Armonk, NY, USA) for data analyses.

Table 2

Description of the instructional differences and identical lesson content for the study groups

\begin{tabular}{|c|c|c|}
\hline Instruction & Experiment group & Comparison group \\
\hline Activity directions & Quick Response (QR) code for activity directions & Verbal instruction for activity directions \\
\hline Task sheet completion & Edmodo: Digital data table & Paper and pencil data table \\
\hline Heart rate calculation & Instant Heart Rate application & Manual calculation \\
\hline Lesson & Topic & Physical activity \\
\hline Lesson one & Relative physical activity intensity & Walk/Jog Talk Test, Fitness Stations \\
\hline Lesson two & Heart rate and physical activity intensity & Walk/Jog \& Talk w/Heart Rate, Fitness Stations \\
\hline Lesson three & Energy expenditure I & Walk/Jog \& Talk w/Heart Rate, Fitness Stations \\
\hline Lesson four & Energy expenditure II & Walk/Jog/Run \& Talk w/Heart Rate, Fitness Stations \\
\hline Lesson five & Energy balance & Walk \& Talk w/Heart Rate, Fitness Stations \\
\hline
\end{tabular}

Note. Both groups used the identical lesson plans. 


\section{Results}

\section{Variable descriptive statistics}

The aggregated average of student situational interest and physical activity variables were listed in Table 3 . Levene's test for equality of variances showed that equal variances of the comparison and experiment group data were assumed for the situational interest scores $(F=0.97, p=.47)$. The Pearson-product moment correlation coefficients between the study variables are presented in Table 4. Based on the pooled sample, MVPA had a strong, positive correlation with the step/min $(r=.79)$, and moderate correlation with energy expenditure $(r=.47)$. A statistically significant, moderate correlation was found between step/min and the amount of energy expenditure $(r=.32)$. Low correlations were found to exist between student interest and step count $(r=.21)$ as well as MVPA $(r=.15)$. Situational interest was not found to be significantly correlated with energy expenditure $(r=-.09)$ or the amount of light physical activity $(r=-.05)$.

\section{Group differences in physical activity and situational interest}

Results from analysis of variance with repeated measures indicated that there was a statistically significant, between-group effect, Pillai's $\lambda=0.65, F=94.51$, $p<.001, \eta^{2}=.65$. The result suggested that students in the experiment group spent a statistically significantly lower amount of time participating in MVPA when compared to the comparison group. Specifically, as shown in Table 5, there was no significant difference in light activity time, but there were statistically significant differences in the step count, amount of energy expenditure, time spent participating in MVPA, and student's situational interest. Students participating in the technology-integrated lessons took a statistically significantly lower amount of steps, averaging approximately 180 fewer steps than students in the comparison group. Students who participated in the comparison group burned an average of 16 more kcals than those students who participated in the technology-integrated physical education lessons. There was no statistically significant difference between groups for light physical activity time.

\section{Physical activity and situational interest fluctuation across lessons}

As shown in Table 5 , there was a statistically significant within-lesson effect, Pillai's $\lambda=.43, F=6.08, p=.01$, $\eta^{2}=.11$ for both groups. The results suggested that there were significant differences among the lessons regarding light physical activity time, steps count, and MVPA. The post hoc tests (Tukey's HSD) suggested

Table 3

Descriptive statistics of the variables across five lessons $(M \pm S D)$

\begin{tabular}{lcc}
\hline Variable & Experiment group & Comparison group \\
\hline Sedentary (min) & $6.54 \pm 1.80$ & $4.12 \pm 1.28$ \\
Light physical activity (min) & $18.27 \pm 3.85$ & $18.46 \pm 3.33$ \\
MVPA (min) & $8.25 \pm 2.79$ & $14.36 \pm 2.67$ \\
Step/min & $29.17 \pm 5.04$ & $34.96 \pm 4.78$ \\
Energy expenditure (kcal) & $30.69 \pm 9.92$ & $49.86 \pm 27.85$ \\
Situational interest & $3.38 \pm 0.92$ & $3.65 \pm 0.87$ \\
\hline
\end{tabular}

Note. $\mathrm{MVPA}=$ moderate-to-vigorous physical activity

Table 4

Pearson product-moment correlation coefficients between the variables

\begin{tabular}{lccccc}
\hline Variable & 1 & 2 & 3 & 4 & 5 \\
\hline 1. Light activity (min) & - & & & & \\
2. MVPA (min) & $-.25^{*}$ & - & & & \\
3. Step/min & $-.17^{*}$ & $.79^{*}$ & - & & \\
4. Energy expenditure (kcal) & .01 & $.47^{*}$ & $.32^{*}$ & - & \\
5. Situational interest & -.05 & $.15^{*}$ & $.21^{*}$ & -.09 & - \\
6. Sedentary (min) & $-.54^{*}$ & $-.68^{*}$ & $-.56^{*}$ & $-.42^{*}$ & -.10 \\
\hline
\end{tabular}

Note. $\quad$ MVPA $=$ moderate-to-vigorous physical activity. $\quad{ }^{*} p<.05$ 
Table 5

Test of group, lesson, and group $\times$ lesson effects on physical activity and interest

\begin{tabular}{|c|c|c|c|c|}
\hline Variable & $M S$ & $F$ & $p$ & Partial $\eta^{2}$ \\
\hline \multicolumn{5}{|l|}{ Group effect } \\
\hline Light physical activity (min) & 2.48 & 0.25 & .62 & $<.001$ \\
\hline Step/min & $2,223.31$ & 145.96 & $<.001$ & .36 \\
\hline Energy expenditure (kcal) & $24,336.63$ & 54.05 & $<.001$ & .18 \\
\hline Situational interest & 4.75 & 5.81 & .02 & .02 \\
\hline MVPA (min) & $2,475.69$ & 389.83 & $<.001$ & .61 \\
\hline \multicolumn{5}{|l|}{ Lesson effect } \\
\hline Light physical activity (min) & 36.91 & 3.74 & .01 & .06 \\
\hline Step/min & $1,899.66$ & 31.18 & $<.001$ & .33 \\
\hline Energy expenditure (kcal) & 323.67 & 0.72 & .58 & .01 \\
\hline Situational interest & 0.05 & 0.06 & .99 & $<.001$ \\
\hline MVPA (min) & 70.07 & 11.03 & $<.001$ & .15 \\
\hline \multicolumn{5}{|l|}{ Group $\times$ lesson interaction } \\
\hline Light physical activity (min) & 182.34 & 18.46 & $<.001$ & .23 \\
\hline Step/min & 141.00 & 9.26 & $<.001$ & .13 \\
\hline Energy expenditure (kcal) & 136.07 & 0.30 & .88 & .01 \\
\hline Situational interest & 0.87 & 1.06 & .38 & .02 \\
\hline MVPA (min) & 16.17 & 2.55 & .04 & .04 \\
\hline
\end{tabular}

Note. $M S=$ mean square; $\mathrm{MVPA}=$ moderate-to-vigorous physical activity.

that there were significant differences in the amount of time spent participating in light physical activity, MVPA, and step count. Significant differences were in light physical activity between lessons 1 and 2 as well as lessons 2 and 4 . Statistically significant differences for the number of steps taken were found between lesson 1 and each of the following 4 physical education lessons, whereas there were no significant differences amongst lessons 2, 3, 4, and 5. The highest amount of light physical activity time occurred during lesson 1. Students reported the lowest amount of time spent participating in light physical activity during lesson 3. The final set of significant differences were in MVPA between lesson 1 and the following 4 physical education lessons. Of the five lessons, lessons 3 and 5 produced the highest amount of time spent participating in MVPA. During lesson 1, students reported the lowest MVPA time. There were no statistically significant differences between the lessons for the variables of energy expenditure or students' situational interest.

As displayed in Table 5, there was a statistically significant group $\times$ lesson interaction effect, Pillai's $\lambda=.40, F=5.59, p=.01, \eta^{2}=.10$. Statistically significant lesson and group interactions occurred between the variables of light physical activity time (Figure 1), step/min (Figure 2), and MVPA time (Figure 3). There were no significant interactions found between the variables of energy expenditure or students' situational interest.

\section{Discussion}

\section{Situational interest difference and fluctuation across the} lessons

Situational interest may change depending upon the context rendered by the instruction and content in physical education. The results indicated that students in the comparison group reported higher situational interest in the physical education lessons than their experiment counterparts. This finding is contrary to Sun (2012) who found that students participating in a technology-integrated Exergame unit reported higher situational interest than they did when participating in a fitness education unit. Technology usage itself might have impacted these two different outcomes. Exergames carried physical activity prompt, forcing students to be engaged in the physical activities. The mobile technologies such as the iPad applications in the current study had no physical activity prompt, 


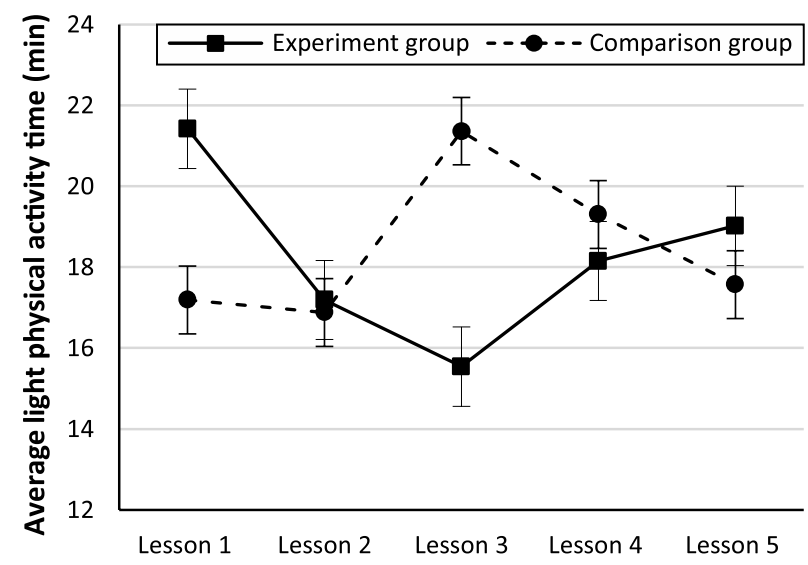

Figure 1. Light physical activity time across the lessons.

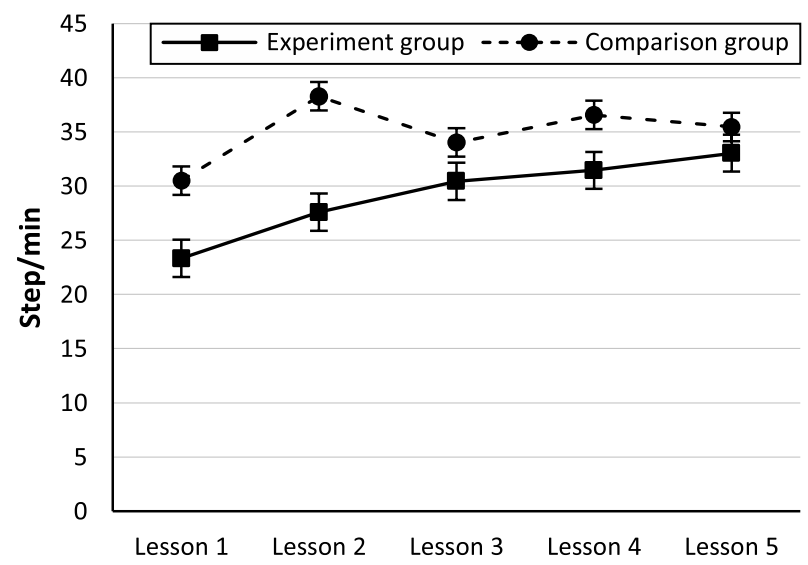

Figure 2. Step/min during the lessons.

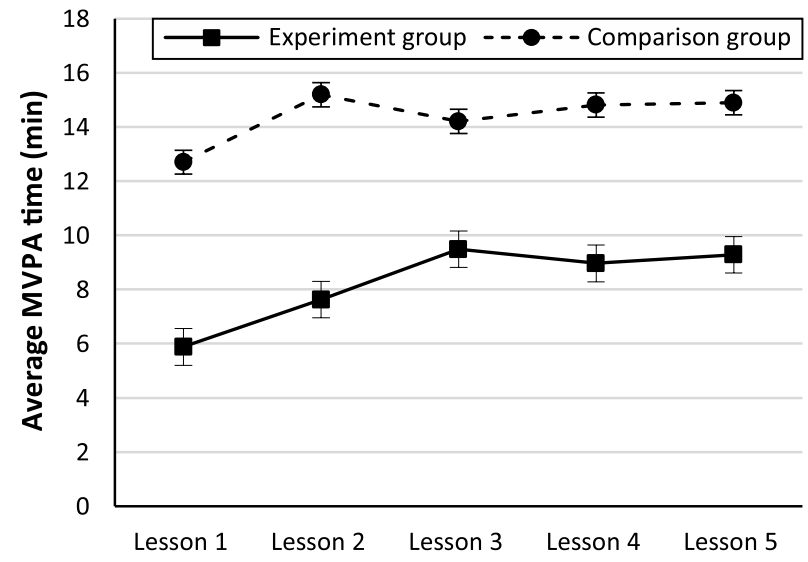

Figure 3. Moderate-to-vigorous physical activity (MVPA) time during the lessons.

merely giving directions/information without requiring little or no physical activity to engage, but instead required cognitive thinking and execution to complete the instructional tasks. As reported by Zhu (2013), for students in middle schools, the first thing that they expect to learn or do in physical education is to have fun participating in physical activities, and they do not expect to be learning the conceptual knowledge. When student expectations are taken into consideration, the results of the current study do not appear as unusual.

The significant differences in student interest for the experiment group were found between lesson 1 and the following four lessons. One explanation for the low level of interest reported during lesson 1 may be that the initial lesson of technology-integration took time away from students participating in what they believed were interesting activities and replaced it with the issuing of iPads and the troubleshooting of problems that occurred with the applications. Similar results were reported by teachers and students in other research (Woods, Karp, Miao, \& Perlman, 2008). During lesson observations, the teachers reported that a substantial amount of time was taken away from activity time due to checking out iPads, logging into applications, troubleshooting problems and security features that limited accessibility. Another explanation for the lower level of interest reported during the initial technologyintegrated lesson, may have been due to a learning curve that is associated with integrating new technology applications and may account for the low level of reported interest (Angers \& Machtmes, 2005; Woods et al., 2008).

Based on anecdotal interaction, the teachers participating in the research lessons believed that technology, when used appropriately, could be a great motivational tool to assist in engaging students in physical education activities in which they may not otherwise have situational interest. However, teachers participating in the research study were also concerned that students often became more interested in the technology than the instructional goal of gaining the conceptual understanding of specific skills. Without multiple direct interviews or other interaction with the students, it remains unclear whether the students may have been more interested in the technology than physical education content; however, it is certainly a valid concern from the teachers' perspective that whatever technological tools they use during instruction should not replace or outweigh the class content and become the focal point of situational interest.

\section{Physical activity difference and fluctuation}

While many studies have reported and compared students' physical activities levels in a variety of physical education lessons (Culpepper, Tarr, \& Killion, 2011; Flohr, Todd, \& Tudor-Locke, 2006; Gao et al., 2010; Strand \& Reeder, 1993), no study has specifically reported the physical activity fluctuation within similar types of technology-integrated lessons. The current study revealed that the step count during the 
technology-integrated physical education lessons increased significantly over the five lesson research period. This increase could have been in part due to students becoming more familiar with the use of the instructional technology and being able to spend more time participating in physical activities instead of troubleshooting the technology-integrated elements of the lesson.

The amount of time spent participating in MVPA in physical education varies depending on lesson content, time spent dressing out, instructional time, and transition time between activities (Chen, Sun, Zhu, \& Ennis, 2012). In this study, the amount of time students spent participating in MVPA in the experiment group ( $8.25 \mathrm{~min}$ ) equated to approximately $28 \%$ of the total time $(\sim 30 \mathrm{~min})$ allotted for physical activity. This result indicated that a majority of students' physical education class time was spent participating in activities below the desirable MVPA threshold (50\%). The low level of time spent participating in MVPA was similar to Sun (2012) who found that Exergaming stations in physical education did not provide adequate MVPA, even though it did spark and maintain students' situational interest in the technology-integrated physical activities.

Although there were significant differences found between the two groups in MVPA, the gap in time spent participating in MVPA narrowed as the students in the experiment group became acclimated with the technology-integrated elements of the physical education lessons. Thus, it appeared that both the teachers and students needed to become more familiar with the technology and applications before the lessons could reach their full potential. Earlier studies have suggested that many physical education teachers felt competent in using several types of technologies (Angers \& Machtmes, 2005; Woods et al., 2008). In this study, it appeared that even with the initial training, after the five lessons it was difficult to tell whether the teachers and students were using the technologies to their fullest potential.

\section{Limitations and implications}

Several limitations should be noted in the study. As shown in the other studies, there might be a learning curve in implementing technologies in the classroom, which would take a longer time to realize the full potential. Second, the sample size is relatively small. Four teachers in particular would not be large enough to represent the spectrum pedagogical factors. Additionally, other factors such as student attitude or general interest might affect their involvement in physical activity during PE which is examined in the study. Finally, no measure of student learning was included in the study, thus we are unable to discern the effect on student learning. Future studies should follow the technology integration for a longer period, with a larger sample size, and/or include a learning variable to provide a more comprehensive investigation. Other factors such as general attitude or interest in PE or physical activity could play a role as well. Research is needed to address the multitude of mobile applications that can be used to track physical activity to determine how effective they are at increasing out of class physical activity after being introduced to students in the classroom.

\section{Conclusions}

In conclusion, neither of our hypotheses was supported by the findings. The data from this preliminary study suggested that mobile technology-integrated physical education might not be effective for increasing students' physical activity levels over a short duration of time, particularly when the mobile technology such as iPad and applications had no direct physical activity prompt. It is important to consider the classroom dynamics to realistically evaluate the strengths and constraints that technology-integrated physical education lessons may pose in a traditional physical education environment. A relatively long learning curve should be expected for both teachers and students to effectively implement mobile technologies to their fullest potential.

\section{Conflict of interest}

There were no conflicts of interest.

\section{References}

Angers, J., \& Machtmes, K. (2005). An ethnographic-case study of beliefs, context factors and practices of teachers integrating technology. Qualitative Report, 10, 771-794.

Casey, A., \& Jones, B. (2011). Using digital technology to enhance student engagement in physical education. AsiaPacific Journal of Health, Sport and Physical Education, 2, 51-66.

Centers for Disease Control and Prevention. (2010). Strategies to improve the quality of physical education. Washington, DC: U.S. Department of Health and Human Services.

Chen, A., Darst, P., \& Pangrazi, R. (1999). What constitutes situational interest? Validating a construct in physical education. Measurement in Physical Education and Exercise Science, 3, 157-180.

Chen, A., Sun, H., Zhu, X., \& Ennis, C. D. (2012). Influence of personal and lesson factors on caloric expenditure in 
physical education. Journal of Sport and Health Science, 1, 49-56.

Culpepper, D. O., Tarr, S. J., \& Killion, L. E. (2011). The role of various curriculum models on physical activity levels. Physical Educator, 68, 163-171.

Flohr, J., Todd, M., \& Tudor-Locke, C. (2006). Pedometerassessed physical activity in young adolescents. Research Quarterly for Exercise and Sport, 77, 309-315.

Freedson, P., Pober, D., \& Janz, K. F. (2005). Calibration of accelerometer output for children. Medicine \& Science for Sports \& Exercise, 37, S523-S530.

Gao, Z., Lee, A. M., Solmon, M. A., Kosma, M., Carson, R. L., Tao, Z., ... More, D. (2010). Validating pedometerbased physical activity time against accelerometer in middle school physical education. ICHPER-SD Journal of Research in Health, Physical Education, Recreation, Sport \& Dance, 5, 20-25.

Hall, T. (2012). Emplotment, embodiment, engagement: Narrative technology in support of physical education, sport and physical activity. Quest, 64, 105-115.

Hänggia, J., Phillips, L., \& Rowlands, A. (2013). Validation of the GT3X ActiGraph in children and comparison with the GT1M ActiGraph. Journal of Science and Medicine in Sport, 16, 40-44.

Hidi, S., \& Renninger, K. (2006). The four-phase model of interest development. Educational Psychologist, 41, 111-127.
Kervin, L., Verenikina, I., Jones, P., \& Beth, O. (2013). Investigating synergies between literacy, technology and classroom practice. Australian Journal of Language and Literacy, 36, 135-147.

National Association for Sport and Physical Education. (2003). What constitutes a quality physical education program [Position statement]. Reston, VA: Author.

Sasaki, J., John, D., \& Freedson, P. (2011). Validation and comparison of ActiGraph activity monitors. Journal of Science and Medicine in Sport, 14, 411-416.

Shen, B., \& Chen, A. (2006). Examining the interrelations among knowledge, interests, and learning strategies. Journal of Teaching in Physical Education, 25, 182-199.

Strand, B., \& Reeder, S. (1993). Analysis of heart rate levels during middle school physical education activities. Journal of Physical Education, Recreation and Dance, 64, 85-91.

Sun, H. (2012). Exergaming impact on physical activity and interest in elementary school children. Research Quarterly for Exercise and Sport, 83, 212-220.

Woods, M., Karp, G., Miao, H., \& Perlman, D. (2008). Physical educators' technology competencies and usage. Physical Educator, 65, 82-99.

Zhu, X. (2013). Exploring students' conception and expectations of achievement in physical education. Measurement in Physical Education and Exercise Science, 17, 62-73. 